FD-02

\section{Variable Temperature Scanning Hall Probe Microscopy (SHPM) Using Quartz Crystal} AFM Feedback.

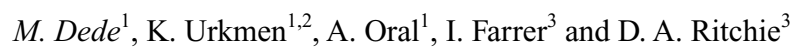

1. Physics, Bilkent University, Ankara, Turkey; 2. NanoMagnetics Instruments Ltd, Oxford, United Kingdom; 3. Cavendish Laboratory, University of Cambridge, Cambridge, United Kingdom

Scanning Hall Probe Microscopy (SHPM)[1] is a quantitative and non-invasive technique for imaging localized surface magnetic field fluctuations such as ferromagnetic domains with high spatial and magnetic field resolution of $\sim 50 \mathrm{~nm}[2]$ and $7 \mathrm{mG} / \sqrt{\mathrm{Hz}}$ at room temperature. In the SHPM technique, Scanning Tunneling Microscope (STM) [1] or Atomic Force Microscope (AFM)[3] feedback is used to keep the Hall sensor in close proximity of the sample. However, STM tracking SHPM requires conductive samples; therefore the insulating substrates have to be coated with a thin layer of gold. This constraint can be eliminated with the AFM feedback using sophisticated Hall probes that are integrated with AFM cantilevers. However it is very difficult to micro fabricate these sensors. In this work, we have eliminated the difficulty in the cantilever-Hall probe integration process, just by gluing a Hall Probe chip to a quartz crystal tuning fork force sensor. The resultant SHPM can operate in variable temperature environment, $77-300 \mathrm{~K}$

The $800 \mathrm{~nm}$ size Hall sensors are microfabricated in a class 100 clean room facility. The Hall sensor chip is simply glued at the end of a $32,768 \mathrm{~Hz}$ Quartz crystal, which is used as force sensor as shown in Fig. 1. An LT-SHPM system is used to scan the samples as shown in Fig. 2. The sensor assembly is dithered at the resonance frequency using a digital Phase Locked Loop circuit and frequency shifts are used for AFM tracking. SHPM electronics is modified to detect AFM topography and the phase, along with the magnetic field image. Magnetic domains and topography of the Iron Garnet thin film crystal, NdFeB demagnetised magnet and hard disk samples are imaged at 300K as shown in Fig 3 with the Quartz Crystal AFM feedback. The system is designed to operate down to $1 \mathrm{~K}$, but it could only be tested down to $77 \mathrm{~K}$ for the moment, as shown in Fig. 4.

In conclusion, the performance is found to be comparable with the SHPM using STM feedback under variable temperature operation. Quartz Crystal AFM feedback offers a very simple sensor fabrication and operation in SHPM. This method eliminates the necessity of conducting samples for SHPM.

[1] A. Oral et. al. Appl. Phys. Lett., 69, 1324 (1996)

[2] A. Sandhu et. al. J. J. Appl. Phys. Vol. 43, No. 2, 777-778 (2004)

[3] A.J. Brook et. al. Appl. Phys. Lett. 82, 3538 (2003)
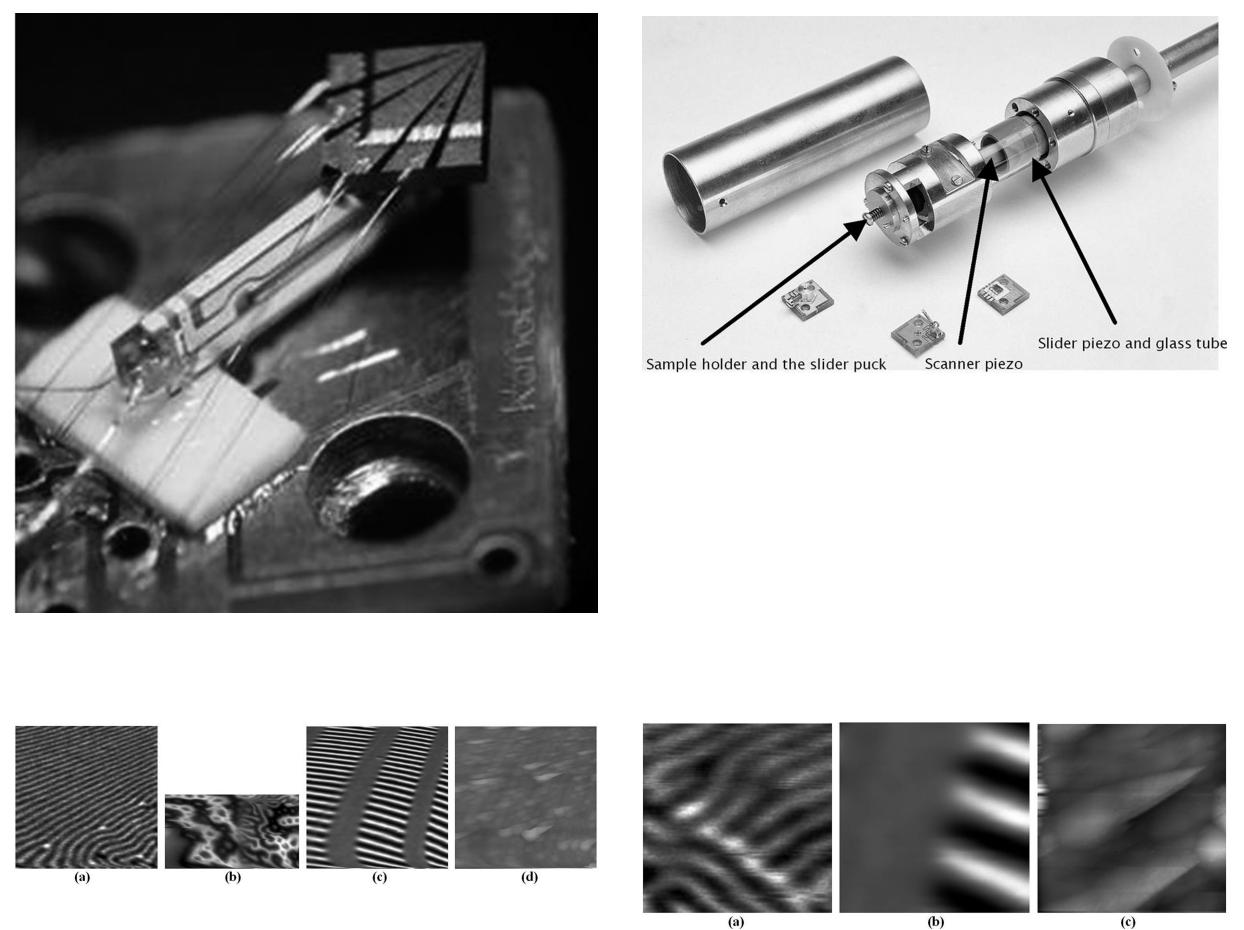